\title{
Tratamento organosolv aplicado ao bagaço de cana para utilização na descontaminação de soluções contendo zinco
}

\author{
Organosolv treatment applied to sugarcane bagasse for use in \\ decontamination solutions containing zinc
}

\author{
1 Bruno Chaboli Gambarato bruno.gambarato@foa.org.br \\ 2 Fernanda Rodrigues da Silva
}

1 Docente do Centro Universitário de Volta Redonda, UniFOA.
2 Mestre em Materiais pelo Centro Universitário de Volta Redonda, UniFOA.

\section{Resumo}

A poluição de águas por metais pesados é um problema que tem afetado a sociedade, principalmente nos últimos 50 anos. Por esse motivo, a legislação preconiza que os efluentes industriais sejam tratados e descontaminados antes de serem liberados nos corpos d'água. Assim, neste trabalho, foi realizada a descontaminação de soluções contendo o metal zinco por meio da utilização do bagaço de cana-de-açúcar. Ao bagaço, foi aplicado um tratamento organosolv, utilizando- se uma mistura de etanol:água, contendo $\mathrm{HCl}$ como catalisador. Os resultados mostraram que o tratamento forneceu um material mais cristalino que o bagaço in natura, sugerindo a remoção de frações amorfas, como os extrativos, as hemicelulose e parte da lignina. 0 mesmo comportamento foi verificado na análise térmica, reforçando os indícios de remoção desses componentes. Tanto o bagaço in natura quanto o bagaço tratado foram capazes de biossorver o zinco, porém os resultados obtidos com a utilização do bagaço tratado foram muito melhores, sugerindo que a remoção das frações amorfas melhorou as propriedades de biossorção de zinco do bagaço de cana.

\section{Palavras-chave}

bagaço de cana; organosolv; biossorção; metais pesados; zinco.

\begin{abstract}
Water pollution by heavy metals is a problem that has affected society especially in the last 50 years. For this reason, law calls for industrial effluents be treated and decontaminated before being released. In this work, the decontamination of solutions containing zinc by biosorption using sugarcane bagasse were evaluated. An organosolv treatment was applied to bagasse using a mixture of ethanol: water containing $\mathrm{HCl}$ as catalyst. The results showed that the treatment provided an increase in crystallinity, suggesting the removal of amorphous fraction, such as extractives, hemicellulose and part of lignin content. The same behavior was observed in thermal analysis, also indicating the removal of these components. Both the bagasse in natura as treated bagasse were able to biossorb zinc, however, the results obtained with the use of treated bagasse were much better, suggesting that the removal of the amorphous fraction improved properties of zinc biosorption of bagasse.
\end{abstract}

\section{Keywords}

sugarcane bagasse; organosolv; biosorption; heavy metals; zinc.

\section{Como você deve citar?}

GAMBARATO, Bruno Chaboli; SILVA, Fernanda Rodrigues da. Tratamento organosolv aplicado ao bagaço de cana para utilização na descontaminação de soluções contendo zinco. Cadernos UniFOA, Volta Redonda, n. 33, p. 37-48, abr. 2017. 


\section{INTRODUÇÃO}

Os metais pesados, em consequência da crescente atividade agrícola e industrial, atingem o solo, o lençol freático e as águas de abastecimento e podem causar danos irreparáveis à saúde da população.

Diversas estratégias de remediação de águas contaminadas com metais pesados têm sido estudadas (RODRIGUES et al, 2016; KARNITZ, 2007; GOLIN, 2007; HAYASHI, 2001). A possibilidade da utilização de resíduos agroindustriais nesse processo desperta interesse da comunidade científica, pois, além da despoluição da água, oferece ainda uma forma de utilização desses resíduos, agregando, assim, mais valor à matéria-prima inicial.

O Brasil é um grande produtor agrícola e, anualmente, são geradas bilhões de toneladas em resíduos sólidos provenientes da agroindústria. A cana-de-açúcar é uma das principais monoculturas nacionais e, somente na safra 2014/2015, o Brasil gerou cerca de 200 milhões de toneladas de resíduos (UNICA, 2016), principalmente bagaço e palha. Isso porque, no processamento da cana para a obtenção de etanol, esses resíduos, hoje denominados subprodutos, são gerados a partir da colheita e da moagem da matéria-prima.

Nas usinas, o bagaço é utilizado, principalmente, para a obtenção de energia, por meio da queima nas caldeiras industriais. Entretanto, com o desenvolvimento da tecnologia nas caldeiras e os crescentes estudos na área de conversão de biomassa, o bagaço de cana pode contribuir com a geração de diversos produtos, além da energia. Entre esses produtos, o principal é o etanol de segunda geração (NEVES et al, 2016; MESA et al, 2016), que oferece à indústria a oportunidade de aumentar a produção de etanol sem aumentar o plantio de cana-de-açúcar (GUBICZA et al, 2016).

Além disso, o bagaço de cana pode fornecer matéria-prima para uma gama de outros produtos, como polpa celulósica (ZHANG et al, 2016), nanocristais de celulose (CAMARGO et al, 2016), reforço em compósitos poliméricos (CARVALHO NETO et al, 2014), xilitol (SILVA et al, 2015; KAMAT et al, 2013) e diversos derivados de lignina, como vanilina e ácido vanílico, resinas fenólicas e matrizes para formulações de liberação controlada (OLIVEIRA e GONÇALVES, 2013a e 2013b).

Dessa forma, com vistas à problemática da poluição das águas com metais pesados e ao aproveitamento do bagaço de cana, este trabalho pretende utilizar esse subproduto na descontaminação de águas contendo zinco por meio da modificação do bagaço via tratamento organosolv.

\section{MATERIAIS E MÉTODOS}

\subsection{Obtenção da biomassa}

O bagaço de cana-de-açúcar utilizado neste trabalho foi obtido na cidade de Canas - SP, diretamente com um produtor. $\mathrm{O}$ bagaço foi recebido, desmedulado e lavado com água até total remoção dos açúcares da cana. Procedeu-se, então, a secagem do material ao sol, durante 24 horas. Em seguida, o material foi moído e armazenado. 


\subsection{Tratamento Químico}

\subsubsection{Pré-tratamento alcalino}

O bagaço de cana-de-açúcar foi submetido a um tratamento alcalino com o objetivo de solubilizar parte da lignina do material, bem como facilitar a etapa posterior de Polpação organosolv. Para tal, cerca de $100 \mathrm{~g}$ de bagaço moído foram colocadas em, aproximadamente, $1 \mathrm{~L}$ de solução contendo 0.1 mol.L-1 de hidróxido de sódio $(\mathrm{NaOH})$ e $0,2 \mathrm{~mol}^{-L^{-1}}$ de hipoclorito de sódio ( $\mathrm{NaHClO}$ ). O bagaço ficou imerso nessa solução em temperatura ambiente durante um período total de 48 horas, sendo, dessas, aproximadamente, 24 horas, ao sol. A exposição à luz do sol se deve ao fato da lignina, que compõe cerca de $25 \%$ do material, apresentar estrutura polifenólica, sendo, portanto, bastante sensível a esse tipo de radiação. Assim, o objetivo da exposição à luz solar é degradar parte da estrutura polifenólica da lignina, já parcialmente sensível em função da solução alcalina.

Ao final do tratamento, o material foi lavado exaustivamente com água e, em seguida, foi seco e armazenado.

\subsubsection{Tratamento organosolv}

O material pré-tratado alcalinamente foi submetido a um processo de polpação organosolv. 0 objetivo desse tratamento é degradar parte da lignina por solvólise e remover parte da hemicelulose do material. Assim, obtêm-se um material mais fibroso, rico em celulose. Para tal, a reação ocorreu em balão reacional de $1 \mathrm{~L}$, contendo $500 \mathrm{~mL}$ de etanol $92 \%, 11 \mathrm{~mL}$ de ácido clorídrico $(\mathrm{HCl})$ a $10 \mathrm{~mol} . \mathrm{L}^{-1}$ e água deionizada para completar o volume de $550 \mathrm{~mL}$ de meio reacional. A reação se processou em manta aquecedora a $80^{\circ} \mathrm{C}$, sob refluxo de um condensador de bolas, durante $1 \mathrm{~h}$. Ao final do processo, o conteúdo da reação foi filtrado e lavado com água deionizada até pH neutro. Em seguida, o material foi seco e armazenado.

\subsection{Ensaio de biossorção}

Como fonte de Zinco, foi utilizado o Sulfato de Zinco hepta-hidratado $\left(\mathrm{ZnSO}_{4} \cdot 7 \mathrm{H}_{2} \mathrm{O}\right)$ PA da marca NumaQuímica. Foi preparada uma solução $87,93 \mathrm{mg} \cdot \mathrm{L}^{-1}$ do $\mathrm{ZnSO}_{4} \cdot 7 \mathrm{H}_{2} \mathrm{O}$ para que a concentração de Zinco atingisse $20 \mathrm{mg}^{-\mathrm{L}^{-1}}$.

0 ensaio de biossorção de zinco foi realizado em recipientes de polipropileno de $80,0 \mathrm{~mL}$. Em cada um deles, foram colocados $50,0 \mathrm{~mL}$ da solução de $\mathrm{ZnSO}_{4}$. $7 \mathrm{H}_{2} \mathrm{O}$ e $0,5 \mathrm{~g}$ (base seca) de bagaço de cana (in natura ou tratado) moído até passar pela peneira de 50 mesh. 0 ensaio procedeu durante $48 \mathrm{~h}$, com amostragem nos tempos de 0, 0,5, 1, 2, 5, 24 e 48h. Ao final de cada tempo, o conteúdo foi filtrado e o líquido encaminhado para análise via Espectrometria de Absorção Atômica.

\subsection{Métodos analíticos}

\subsubsection{Difratometria de Raios X}

A difratometria de Raios $\mathrm{X}$ foi realizada com o objetivo de determinar o percentual de cristalinidade do bagaço in natura e do bagaço tratado. Assim, pode-se inferir sobre as alterações estruturais provocadas pelo tratamento químico. Os difratogramas foram obtidos em um difratômetro de Raios $\mathrm{X}$ da marca Shimadzu, modelo XRD 6100, com fonte de radiação CuKa, e voltagem de $40 \mathrm{kV}$, corrente de $40 \mathrm{~mA}$, varredura $0,05(2 \theta / 5 \mathrm{~s})$ para valores de $2 \theta$ entre 10 e $50^{\circ}$. 


\subsubsection{Análise Termogravimétrica}

Com o objetivo de avaliar a estabilidade térmica do material, determinar os parâmetros de degradação térmica e caracterizar modificações estruturais antes e depois do tratamento químico, a Análise Termogravimétrica (TGA) foi realizada em um equipamento Perkin Elmer STA 6000 (Figura 3.4), utilizando-se uma taxa de aquecimento de $10^{\circ} \mathrm{C} \cdot \mathrm{min}^{-1}$, com fluxo de Nitrogênio de $20 \mathrm{~mL}$. $\mathrm{min}^{-1}$, numa faixa de temperatura de 30 a $800^{\circ} \mathrm{C}$.

\subsubsection{Espectrometria de Absorção Atômica}

As determinações das concentrações de zinco foram realizadas via Espectrometria de Absorção Atômica. Para tal, as análises foram realizadas em triplicata em um espectrômetro de absorção atômica Perkin Elmer AAnalyst 800, com forno de grafite.

\section{RESULTADOS E DISCUSSÃO}

\subsection{Caracterização do Bagaço}

As características estruturais do bagaço de cana e as modificações causadas pelo tratamento foram avaliadas por meio das análises de Difratometria de Raios X (DRX) e a Análise Termogravimétrica (TGA).

\subsubsection{Difratometria de Raios $\mathrm{X}$}

Os difratogramas de Raios X do bagaço de cana in natura e do bagaço tratado são mostrados na Figura 1. É possível verificar que os materiais apresentaram, tanto antes quando após o tratamento, um comportamento de materiais semicristalinos, ou seja, que apresentam regiões amorfas e cristalinas em sua estrutura. A determinação do grau de cristalinidade foi realizada pelo método empírico de SEGAL et al (1959), que relaciona, por meio da Equação abaixo, o pico de intensidade da difração do material cristalino - perto de $2 \theta=22^{\circ}$ - com o pico de intensidade da difração do material amorfo - perto de $2 \theta=16^{\circ}$.

$$
\begin{gathered}
I_{c}=\frac{I_{(002)}-I_{(\text {am })}}{I_{(002)}} \times 100 \\
\text { Onde: } \\
\mathrm{I}_{\mathrm{C}}-\text { Índice ou grau de cristalinidade percentual; } \\
\left.\mathrm{I}_{(002)}-\text { Pico de intensidade da difração que representa a fração cristalina (perto de } 2 \theta=22^{\circ}\right) \\
\left.\mathrm{I}_{(\mathrm{am})}-\text { Pico de intensidade da difração que representa a fração amorfa (perto de } 2 \theta=16^{\circ}\right)
\end{gathered}
$$


Figura 1 - Difratogramas de Raios X do bagaço in natura e do bagaço tratado

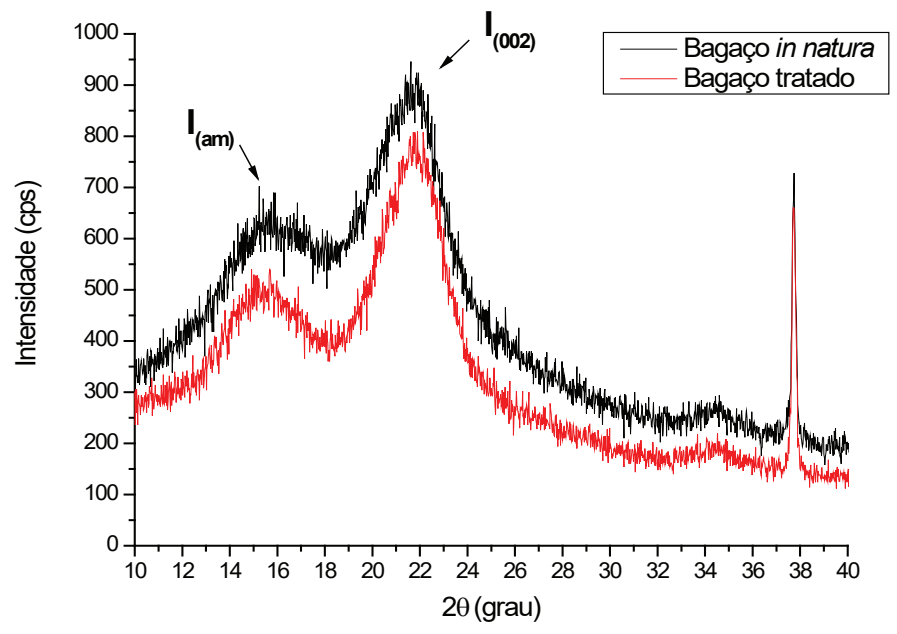

Fonte: dos autores.

A cristalinidade dos materiais foi calculada e os resultados encontram-se na Tabela 1.

Tabela 1 - Parâmetros de cristalinidade dos materiais

\begin{tabular}{llll}
\hline Material & $\mathrm{I}_{(002)}$ & $\mathrm{I}_{(\mathrm{am})}$ & $\mathrm{I}_{\mathrm{c}}(\%)$ \\
Bagaço in natura & 890 & 642 & 27.9 \\
Bagaço tratado & 762 & 478 & 37.3 \\
\hline \multicolumn{4}{c}{ Fonte: dos autores. }
\end{tabular}

Percebe-se que o material in natura possui $27,9 \%$ de cristalinidade, enquanto que, após o tratamento, o material apresentou-se com uma fração cristalina de $37,3 \%$. Sabe-se que, uma vez que o bagaço de cana é um material lignocelulósico e, portanto, composto principalmente de celulose, hemicelulose e lignina, a maior contribuição à fração cristalina provém da celulose, que tem sua cristalinidade garantida pela presença de ligações de hidrogênio intra e intermoleculares. No caso da hemicelulose e da lignina, são macromoléculas altamente amorfas (HENRIKSSON e LENNHOLM, 2009). Dessa forma, o aumento no grau de cristalinidade após o tratamento sugere que, durante esse processo, parte da hemicelulose e da lignina foram removidas do material. É mais provável que a remoção de hemicelulose tenha sido maior, uma vez que essa macromolécula é bastante suscetível a ataques ácidos, enquanto que a lignina é mais resistente a tais reações (FENGEL e WEGENER, 1989).

Assim, é possível inferir que o bagaço tratado pelo processo organosolv se apresenta com um maior teor de celulose e lignina do que o material in natura. Nessas condições, além da aplicação na biossorção de metais, o bagaço pode ser utilizado na produção de etanol de segunda geração (MESA et al, 2016; NEVES et al, 2016) e no desenvolvimento de produtos derivados de celulose, como nanopartículas de celulose (GUBICZA et al, 2016).

\subsubsection{Análise Termogravimétrica (TGA)}

A análise termogravimétrica (TGA) e sua derivada (DTG) foram utilizadas para inferir sobre características estruturais e sobre a composição dos materiais antes e após o tratamento. A curva termogravimétrica obtida para o bagaço in natura e sua primeira derivada são mostradas na Figura 2 , 
onde é possível verificar que ocorre, inicialmente, uma perda de massa até cerca de $100^{\circ} \mathrm{C}$. Essa etapa representa a perda de água pelo material e é inerente a todas as biomassas vegetais, que, mesmo após secagem em estufa, ainda armazenam água em suas estruturas. Em seguida, ocorre entre 120 e $220^{\circ} \mathrm{C}$ uma perda de massa de 6,8\%. Essa etapa está associada à termodegradação dos extrativos presentes no material, da hemicelulose e de compostos de baixa massa molar (GAMBARATO, 2014).

Outra etapa de termodegradação ocorre entre 220 e $320^{\circ} \mathrm{C}$. Nessa faixa de temperatura ocorre a degradação da hemicelulose e de parte da celulose do material (LAURICHESE; AVEROUS, 2014) e a perda de massa corresponde a 31,1\%. A próxima etapa de termodegradação ocorre na faixa de 320 e $380^{\circ} \mathrm{C}$. Nesse intervalo de temperatura, ocorre a degradação da fração mais resistente de celulose e da lignina (GAMBARATO, 2014), correspondendo a $31,8 \%$ do material. Ainda nessa faixa de temperatura, é possível verificar na curva de DTG a temperatura, onde ocorre a máxima degradação do material, $349^{\circ} \mathrm{C}$. Nessa temperatura, a velocidade de termodegradação é máxima. A partir de $380^{\circ} \mathrm{C}$ ocorre a degradação da fração final lignina e o carvão residual obtido em $800^{\circ} \mathrm{C}$ representa $10,82 \%$ do material.

Figura 2 - Curva de termogravimetria para o bagaço in natura e sua primeira derivada

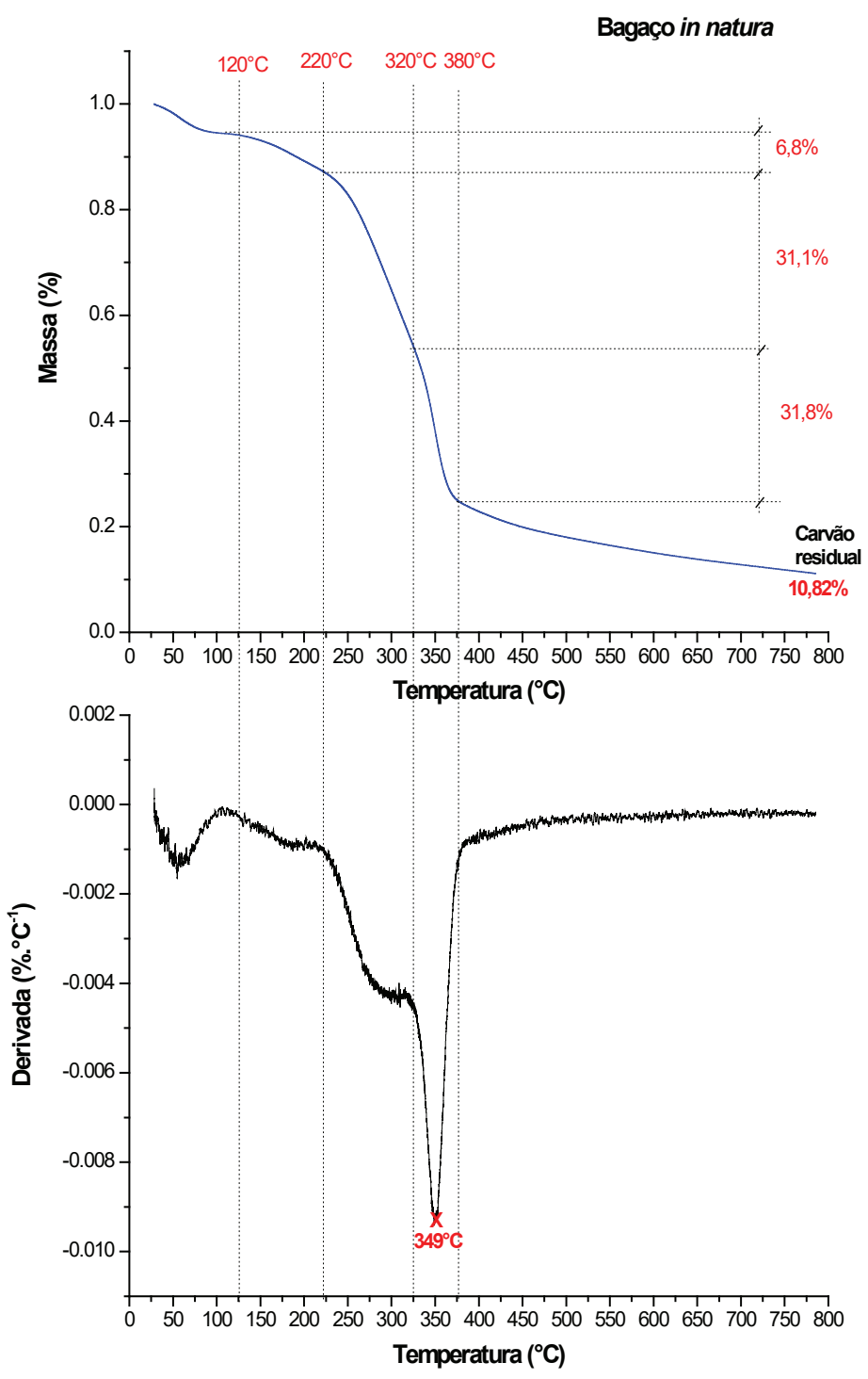

Fonte: dos autores. 
A Figura 3 mostra a curva termogravimétrica para o bagaço tratado e sua derivada. Assim como para o bagaço in natura, é possível verificar a perda inicial de água até a temperatura de cerca de $100^{\circ} \mathrm{C}$. A próxima etapa de termodegradação ocorre na faixa de 180 a $245^{\circ} \mathrm{C}$, representando uma perda de massa de $5,0 \%$, diferente do que ocorre com o bagaço in natura $\left(120\right.$ a $220^{\circ} \mathrm{C}$, perda de massa de $\left.6,8 \%\right)$. Essa é a primeira evidência da remoção de hemicelulose pelo tratamento químico e a Figura 4 exibe uma ampliação dessas faixas de temperatura para melhor comparação.

Figura 3 - Curva de termogravimetria para o bagaço tratado e sua primeira derivada

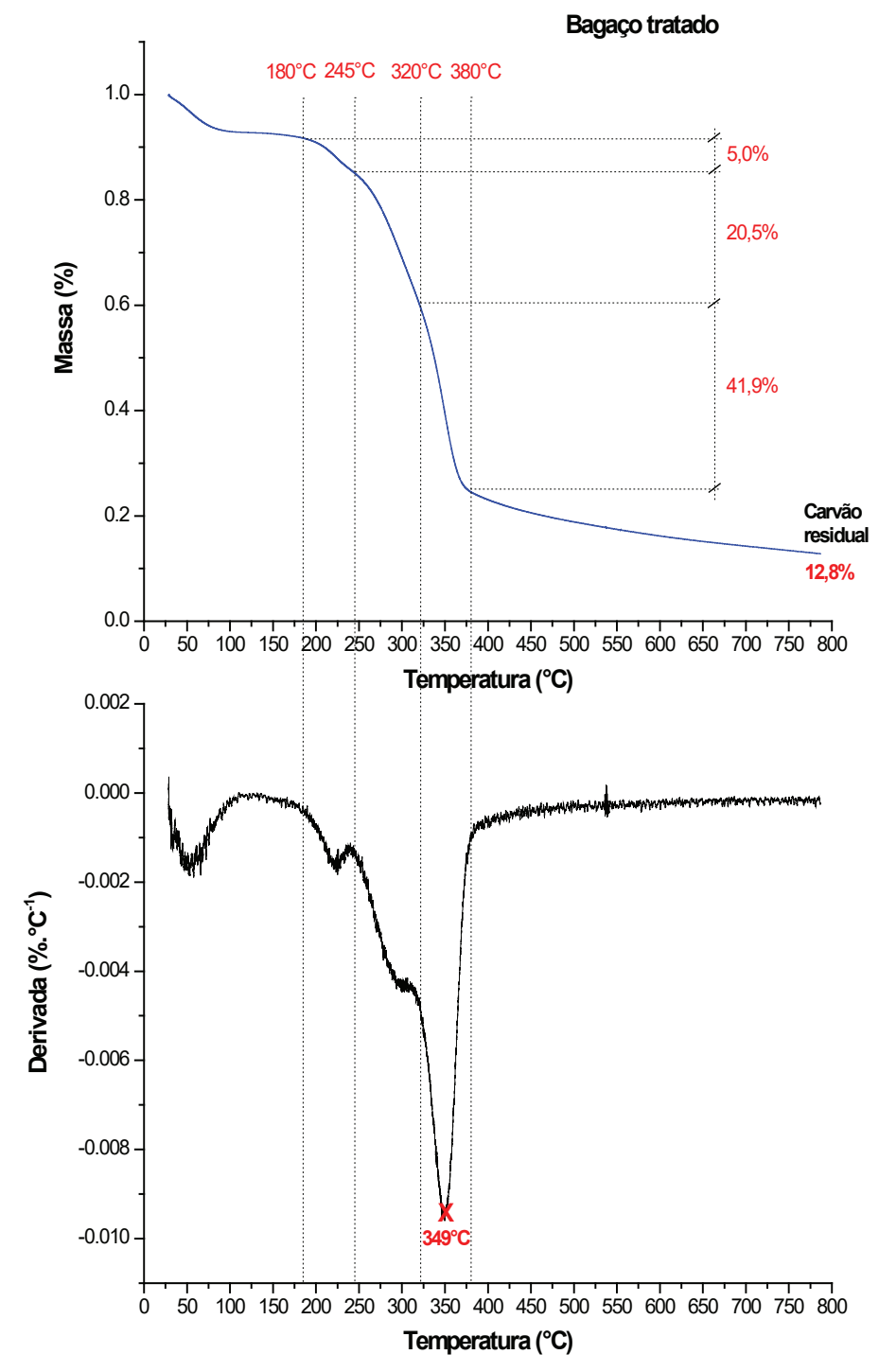

Fonte: dos autores.

O fato da termodegradação do bagaço tratado ocorrer a uma faixa de temperatura maior indica que esse material contém um teor reduzido de hemicelulose e, consequentemente, um teor mais elevado de celulose e lignina. É possível verificar a remoção de hemicelulose também na etapa de termodegradação que ocorre no bagaço tratado na faixa de 245 a $320^{\circ} \mathrm{C}$, que representa $20,5 \%$ em massa do material, enquanto que para o bagaço in natura, essa etapa apresenta uma perda de massa de $30,1 \%$. 
Figura 4 - Curvas de termodegradação do bagaço in natura e do bagaço tratado na faixa de 20 a $320^{\circ} \mathrm{C}$.

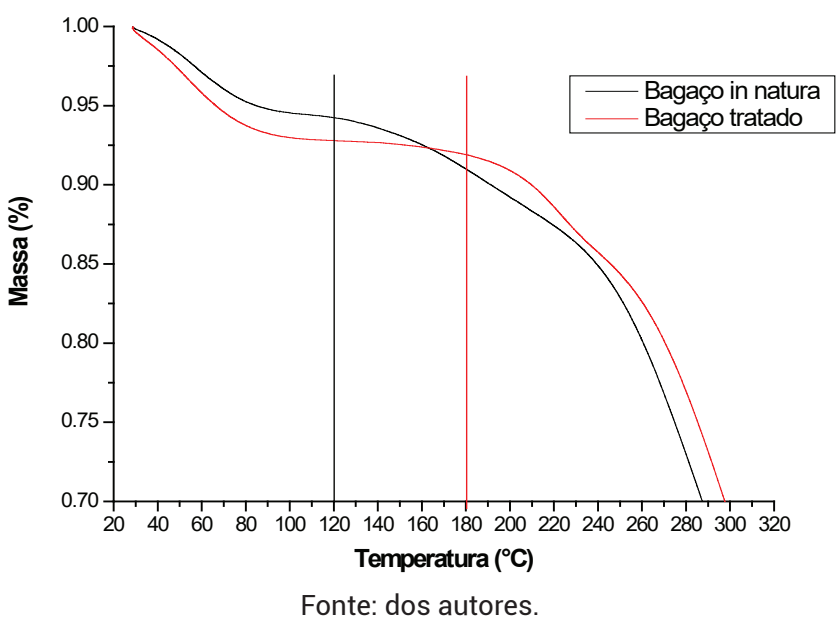

0 maior teor de celulose e lignina apresentado pelo bagaço tratado é evidenciado na etapa de termodegradação que ocorre entre 320 e $380^{\circ} \mathrm{C}$ e representa $41,9 \%$ em massa, valor bastante superior ao apresentado pelo bagaço in natura. 0 carvão residual obtido a $800^{\circ} \mathrm{C}(12,8 \%)$ também se mostrou maior que para o bagaço in natura. Isso porque o maior teor de lignina contribui para esse parâmetro. A temperatura onde ocorreu a máxima degradação térmica foi a mesma encontrada para o bagaço in natura, $349^{\circ} \mathrm{C}$. Tal fato sugere que não houve alterações estruturais importantes na molécula de lignina, sugerindo que o tratamento químico não degrada consideravelmente a lignina do material.

\subsection{Biossorção de Zinco}

A remoção de zinco das soluções contaminadas apresentou melhor desempenho com a utilização do bagaço tratado. Tal comportamento é mostrado na Figura 4.6. Percebe-se que, partindo de uma solução com concentração inicial de $20,04 \mathrm{mg}^{-\mathrm{L}^{-1}}$ de Zinco, o bagaço tratado conseguiu remover metade dessa quantidade antes das 5 primeiras horas de tratamento, enquanto que o tratamento com o bagaço in natura reduziu a concentração à metade da inicial após cerca de 15 horas de tratamento.

Figura 5 - Remoção de zinco das soluções com bagaço de cana in natura e tratado

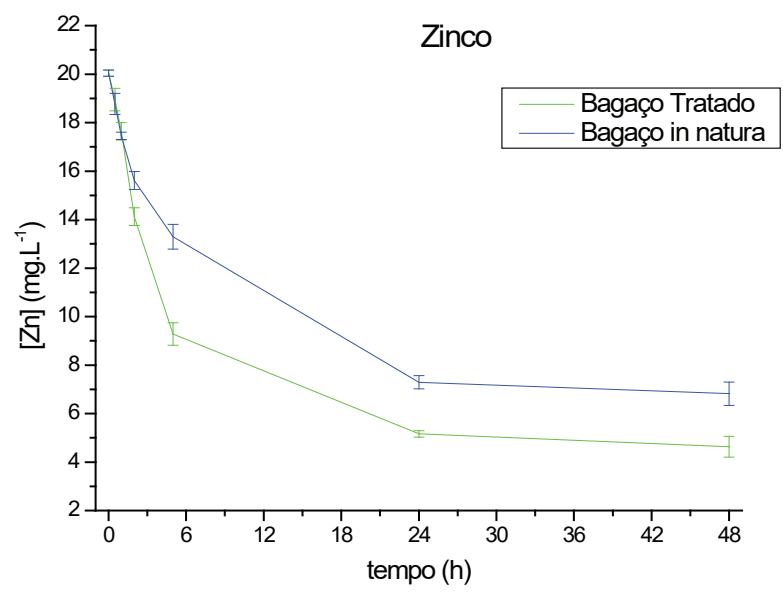

Fonte: dos autores.

Tomando como base o limite estabelecido pela Resolução CONAMA 430/2011 (BRASIL, 2011), a concentração de Zinco não pode ultrapassar $5 \mathrm{mg} \cdot \mathrm{L}^{-1}$. Analisando os tratamentos, percebe-se que 0 
bagaço tratado atinge tal concentração, logo após as primeiras 24 horas de tratamento (Concentração ${ }_{24 h}$ $\left.=5,16 \mathrm{mg}^{\mathrm{L}} \mathrm{L}^{-1}\right)$, enquanto que o bagaço in natura não atinge tal nível, mesmo após as 48 horas.

\section{CONCLUSÁo}

A partir dos resultados obtidos, é possível concluir que:

O tratamento organosolv foi capaz de remover os extrativos, grande parte das hemiceluloses e parte da lignina do material. Tal comportamento foi verificado pelo aumento da cristalinidade após o tratamento, bem como pela modificação das faixas de temperaturas de degradação do material;

O bagaço de cana possui propriedades biossorventes para o zinco. 0 tratamento organosolv aplicado ao material mostrou-se eficiente em aumentar a capacidade biossorvente do bagaço, fato que justifica sua utilização;

\section{REFERÊNCIAS}

BRASIL. Conselho Nacional do Meio Ambiente. Resolução $n^{\circ}$ 430, de 13 de maio de 2011 . Dispõe sobre as condições e padrões de lançamento de efluentes, complementa e altera a Resolução no 357, de 17 de março de 2005. Brasília, 2011. Disponível em: <http://www.mma.gov.br/port/conama/res/res11/ res43011.pdf $>$. Acesso em: 10 maio 2016.

CAMARGO, L. A., PEREIRA, S. C., CORREA, A. C., FARINAS, C. S., MARCONCINI, J. M., MATTOSO, L. H. C. Feasibility of Manufacturing Cellulose Nanocrystals from the Solid Residues of Second-Generation Ethanol Production from Sugarcane Bagasse. BioEnergy Research, p. 1-13, 2016.

CARVALHO NETO, A. G. V., GANZERLI, T. A., CARDOZO, A. L., FÁVARO, S. L., PEREIRA, A. G. B., GIROTTO, E. M., RADOVANOVIC, E. Development of composites based on recycled polyethylene/sugarcane bagasse fibers. Polymer Composites, v. 35, n.4, p, 768-774, 2014.

FENGEL, D.; WEGENER, G. Wood - Chemistry, Ultrastructure, Reactions. Berlin: Walter de Gruyter, 1989.

GAMBARATO, B. C. Isolamento e caracterização de ligninas de palha de cana de açúcar. Tese (Doutorado em Ciências). Universidade de São Paulo. 2014.

GOLIN, D.M. Remoção de chumbo de meios líquidos através de adsorção utilizando carvão ativado de origem vegetal e resíduos vegetais. Dissertação (Mestrado em Engenharia Ambiental). Universidade Federal do Paraná, 2007.

GUBICZA, K.; NIEVES, I. U.; SAGUES, W. J.; BARTA, Z.; SHANMUGAM, K. T.; INGRAM, L. O. Techno-economic analysis of ethanol production from sugarcane bagasse using a Liquefaction plus Simultaneous Saccharification and co-Fermentation process. Bioresource technology, v. 208, p. 42-48, 2016.

HAYASHI, A. M., Remoção de Cromo Hexavalente Através de Processos de Biossorção em Algas Marinhas, Tese (Doutorado em Ciências), UNICAMP, 2001.

HENRIKSSON, G.; LENNHOLM, H. Cellulose and Carbohydrate Chemistry. In EK, M.; GELLERSTEDT, G.; HENRIKSSON, G. Wood chemistry and wood biotechnology. Berlin: Walter de Gruyter, v. 1, p. 71-100, 2009 
KAMAT, S., KHOT, M., ZINJARDE, S., RAVIKUMAR, A., GADE, W. N. Coupled production of single cell oil as biodiesel feedstock, xylitol and xylanase from sugarcane bagasse in a biorefinery concept using fungi from the tropical mangrove wetlands. Bioresource technology, v. 135, p. 246-25, 2013.

KARNITZ, O. Modificação química do bagaço de cana de açúcar e celulose usando anidrido do EDTA. Uso destes materiais na adsorção de metais pesados em solução aquosa. Dissertação (Mestrado em Recursos Hídricos). Universidade Federal de Ouro Preto, 2007.

LAURICHESSE, S.; AVÉROUS, L. Chemical modification of lignins: Towards biobased polymers. Progress in Polymer Science, v. 39, p. 1266-1290, 2014.

MESA, L.; LÓPEZ, N.; CARA, C.; CASTRO, E.; GONZÁLEZ, E.; MUSSATTO, S. I. Techno-economic evaluation of strategies based on two steps organosolv pretreatment and enzymatic hydrolysis of sugarcane bagasse for ethanol production. Renewable Energy, v. 86, p. 270-279, 2016.

NEVES, P. V.; PITARELO, A. P.; RAMOS, L. P. Production of cellulosic ethanol from sugarcane bagasse by steam explosion: Effect of extractives content, acid catalysis and different fermentation technologies. Bioresource technology, v. 208, p. 184-194, 2016.

OLIVEIRA, F. C.; GONCALVES, A. R. . Evaluation of Oxidized Lignin from Coffee Husk on the Obtainment of Added-Value Low-Molecular Weight Compounds. In: XXXIV International Conference on Energy, Biomass and Waste Engineering, v. 76, p. 2776-2781, 2013a.

OLIVEIRA, F. C.; GONCALVES, A. R. Oxidized Sugarcane Bagasse and Coffee Husk Lignin as Compatibilizing Agent for Natural Fiber Reinforced Thermoplastic Composites. In: 21 st European Biomass Conference and Exhibition, p. 1570-1577, 2013b.

RODRIGUES, A.C.D.; SANTOS, A.M.; SANTOS, F.S.; PEREIRA, A.C.C.; SOBRINHO, N.M.B.A. Mecanismos de respostas das plantas à poluição por metais pesados: Possibilidade de uso de macrófitas para remediação de ambientes aquáticos contaminados. Revista Virtual de Química, v.8, n.1, 262-276, 2016.

SEGAL, L. et al. An empirical method for estimating the degree of crystallinity of native cellulose using the X-ray diffractometer. Textile Research Journal, v. 29, p. 786-794, 1959.

SILVA, D. D. V., ARRUDA, P. V., VICENTE, F. M. C. F., SENE, L., SILVA, S. S., Felipe, M. D. G. A. Evaluation of fermentative potential of Kluyveromyces marxianus ATCC 36907 in cellulosic and hemicellulosic sugarcane bagasse hydrolysates on xylitol and ethanol production. Annals of Microbiology, v. 65, n.2, p. $687-694,2015$.

UNICA, União da Indústria de Cana-de-açúcar. Relatório Final da Safra 2014/2015. Disponível em: $<$ www.unicadata.com.br>. Acesso em: 10 maio 2016.

ZHANG, K., SUN, P., LIU, H., SHANG, S., SONG, J., WANG, D. Extraction and comparison of carboxylated cellulose nanocrystals from bleached sugarcane bagasse pulp using two different oxidation methods. Carbohydrate Polymers, v. 138, p. 237-243, 2016. 
Ciências

Sociais

Aplicadas e

Humanas

Applied Social Sciences and Humanities 
